\title{
Gestione clinica del paziente con ipocalcemia
}

\author{
Federica Saponaro ${ }^{1}$
}

Accettato: 22 febbraio 2021 / Pubblicato online: 31 maggio 2021

(c) The Author(s) 2021

\section{Introduzione e fisiopatologia}

Per ipocalcemia si intende un'alterazione biochimica caratterizzata dalla presenza di livelli di calcio sierico ridotti (inferiori a $8,5 \mathrm{mg} / \mathrm{dl}$ o $2,12 \mathrm{mmol} / \mathrm{l}$ ) o livelli di calcio ionizzato ridotto (inferiore a $4,5 \mathrm{mg} / \mathrm{dl}$ o $1,12 \mathrm{mmol} / \mathrm{l}$ ), in presenza di normali concentrazioni di proteine plasmatiche [1]. Si tratta di una condizione di non raro riscontro, che può presentarsi clinicamente come uno spettro che varia da un quadro completamente asintomatico, nei casi più lievi, fino ad un quadro estremamente grave, sintomatico, che rappresenta un' urgenza endocrinologica [2].

Il calcio sierico circolante si trova per circa la metà complessato alle proteine plasmatiche, per una piccola quota $(10 \%)$ complessato in forma salina e per la restante quota come calcio ionizzato libero, che è biologicamente attivo ed essenziale per il normale svolgimento delle funzioni cellulari. I livelli di calcio ionizzato sierico vengono fisiologicamente mantenuti entro un range ristretto e costante grazie all' azione ormonale coordinata di paratormone (PTH), vitamina $\mathrm{D}$ (calcitriolo o $\left.25(\mathrm{OH})_{2} \mathrm{D}\right) \mathrm{e}$, indirettamente, fattore di crescita dei fibroblasti 23 (FGF23). L'azione di questi ormoni si esplica a livello degli organi bersaglio: intestino, rene e osso. Una condizione di ipocalcemia si verificherà in tutti i casi in cui sarà presente una ridotta secrezione del PTH e del calcitriolo o una resistenza periferica a tali ormoni, tale da superare i meccanismi di fisiologico compenso.

Le principali cause di ipocalcemia sono riassunte nella Tabella 1.

$凶$ F. Saponaro

federica.saponaro@unipi.it

1 Dipartimento di Patologia Chirurgica, Medica, Molecolare e dell'Area Critica, Università di Pisa, Pisa, Italia
Tabella 1 Cause di ipocalcemia

- Deficit di vitamina D o resistenza alla vitamina D

- Ipoparatiroidismo post-chirurgico

- Ipoparatiroidismo da cause autoimmuni o genetiche

- Insufficienza renale cronica avanzata

- Pseudoipoparatiroidismo o pseudo-pseudoipoparatiroidismo

- Infiltrazione metastatica o da metalli delle paratiroidi

- Ipomagnesiemia o ipermagnesiemia

- Hungry bone syndrome post-PTx

- Infusione di fosfato o trasfusioni ematiche

- Malattie critiche

- Farmaci (es. bisfosfonati e.v.)

- Sindrome di Fanconi

\section{Presentazione clinica e valutazione}

L'ipocalcemia grave a insorgenza acuta esita più spesso in un quadro clinico severo, che richiede l'ospedalizzazione immediata. In questo caso si parla di crisi ipocalcemica e i sintomi sono il risultato dell'alterazione dell'omeostasi calcica a livello cellulare, con conseguente ipereccitabilità neuro-muscolare. All'esordio si possono avere parestesie degli arti o periorali, fino all'acme della crisi tetanica con: spasmo della muscolatura, contrazione metacarpo-falangea spontanea e dell'avambraccio sul braccio, laringospasmo, broncospasmo, convulsioni accompagnate da bradicardia, ipotensione, aritmie cardiache. La crisi tetanica può essere scatenata o aggravata da farmaci ipocalcemizzanti, condizioni di alcalosi metabolica e dall'iperventilazione. È necessario riconoscere anche i pazienti che presentano sintomi atipici come nausea, vomito, dolore addominale, e fare attenzione ai bambini, nei quali si hanno più spesso crisi convulsive, rispetto ai classici spasmi muscolari [3]. 
Tabella 2 Esami da eseguire in caso di ipocalcemia

Primo Livello
- Calcio sierico (corretto per albumina)
- Fosfato
- Magnesio
- Elettroliti
- Creatinina
- ALP
- PTH
- $25 O H D$
- PH
- Esame emocromo citometrico con formula
Secondo Livello
- Calcio ionizzato
- Calciuria, fosfaturia, magnesiuria delle 24 ore
- Clearance della creatinina
- Ecografia renale (per nefrolitiasi)
- Ricerca di mutazioni genetiche in caso di sospetto di malattia
genetica
- Controllo biochimico dei familiari di primo grado

Nelle forme di ipocalcemia lieve, in genere croniche, la sintomatologia è più sfumata e comprende alterazioni neurologiche (alterazioni cognitivo-comportamentali, disturbi extrapiramidali, calcificazioni intracraniche), alterazioni cutanee o della dentizione e la presenza di ipereccitabilità neuromuscolare latente, che si può svelare evocando i segni di Chvostek e Trousseau.

Per l'appropriato inquadramento anamnestico, è essenziale la storia familiare di alterazioni del metabolismo fosfocalcico e la presenza di ritardo cognitivo o dello sviluppo per sospettare forme genetiche o idiopatiche. Un pregresso intervento sul collo suggerisce, invece, fortemente la diagnosi di ipoparatiroidismo post-chirurgico. All'esame obiettivo sono da ricercare bassa statura, facies lunare, brevità delle ossa metacarpali nel sospetto di pseudoipoparatiroidismo, cicatrici cervicali per l'ipoparatiroidismo post-chirurgico, alopecia, vitiligo e candidiasi mucocutanea per le forme di SPA1.

Nella Tabella 2 sono riportati gli esami di laboratorio e le indagini ulteriori, utili per la diagnosi e la diagnosi differenziale di ipocalcemia.

\section{Terapia}

Nel caso di ipocalcemia acuta e grave, l'obiettivo immediato è controllare la sintomatologia e ristabilire l'equilibro fosfo-calcico. Per valori di calcemia corretta per albumina $<7,5 \mathrm{mg} / \mathrm{dl}(1,88 \mathrm{mmol} / \mathrm{l})$, è necessaria la somministrazione endovenosa di calcio, da preferire con catetere centrale venoso per evitare stravasi e conseguente possibile necrosi tissutale. Le due formulazioni utilizzate sono calcio gluco- nato (94 mg di calcio elementare per grammo) oppure calcio cloruro (272 mg di calcio elementare per grammo), quest'ultimo meno utilizzato perché meno tollerato. La somministrazione immediata prevede calcio gluconato (1-2 fiale da $1 \mathrm{~g} / 10 \mathrm{ml}$ ) in glucosata al 5\% in bolo in 5-10 minuti, per ottenere un incremento del calcio ionizzato in circa 2 ore; successivamente si prosegue con l'infusione lenta continua (a una velocità di circa $0,5 \mathrm{mg} / \mathrm{kg} / \mathrm{h}$ di calcio elementare). Se coesiste ipomagnesiemia, questa deve essere prontamente corretta. Calcio ionizzato e potassiemia devono essere monitorati ogni 4-6 ore, per tutta la durata dell'infusione. L'obiettivo è quello di embricare appena possibile la terapia orale con quella endovenosa, che potrà dunque essere sospesa, al raggiungimento di valori di calcemia corretta per albumina $>7,5 \mathrm{mg} / \mathrm{dl}(1,88 \mathrm{mmol} / \mathrm{l})$ o comunque alla scomparsa dei sintomi [4].

La terapia orale si basa sull'utilizzo di calcio (in genere calcio carbonato $500 \mathrm{mg}=200 \mathrm{mg}$ di calcio elementare, fino a un dosaggio massimo giornaliero di $3 \mathrm{~g} / \mathrm{die}$, refratto in due o tre dosi) e analoghi attivi della vitamina D (preferibilmente calcitriolo $0,5-2 \mu \mathrm{g} / \mathrm{die}$, in dosi refratte). L'obiettivo della terapia orale è mantenere i livelli di calcio sierico corretto per albumina ai limiti inferiori della norma, per evitare l'insorgenza di ipercalciuria e delle sue complicanze.

Nel caso specifico di ipocalcemia da ipoparatiroidismo, la terapia orale con calcio e analoghi della vitamina $\mathrm{D}$, pur riportando i valori di calcemia nel range della norma, non ristabilisce tutte le fisiologiche funzioni del PTH. La diponibilità dal 2015 della terapia sostitutiva con PTH umano ricombinante [rhPTH(1-34) e più recentemente $\operatorname{rhPTH}(1-84)]$, in caso di controllo non ottimale della malattia in terapia convenzionale, apre nuovi orizzonti per i pazienti affetti da questa patologia [5].

Funding Note Open access funding provided by Università di Pisa within the CRUI-CARE Agreement.

Conflitto di interesse L'autrice Federica Saponaro dichiara di non avere conflitti di interesse.

Consenso informato Lo studio presentato in questo articolo non ha richiesto sperimentazione umana.

Studi sugli animali L'autrice di questo articolo non ha eseguito studi sugli animali.

Nota della casa editrice Springer Nature rimane neutrale in riguardo alle rivendicazioni giurisdizionali nelle mappe pubblicate e nelle affiliazioni istituzionali.

Open Access This article is licensed under a Creative Commons Attribution 4.0 International License, which permits use, sharing, adaptation, distribution and reproduction in any medium or format, as long as you give appropriate credit to the original author(s) and the source, provide a link to the Creative Commons licence, and indicate if changes were made. The images or other third party material in this article are included in the article's Creative Commons licence, unless indicated otherwise in a credit line to the material. If material is not included 
in the article's Creative Commons licence and your intended use is not permitted by statutory regulation or exceeds the permitted use, you will need to obtain permission directly from the copyright holder. To view a copy of this licence, visit http://creativecommons.org/licenses/by/4.0/.

\section{Bibliografia}

1. Cooper MS, Gittoes NJ (2008) Diagnosis and management of hypocalcaemia. BMJ 336(7656):1298-1302
2. Fong J, Khan A (2012) Hypocalcemia: updates in diagnosis and management for primary care. Can Fam Physician 58(2):158-162

3. Schafer AL, Shoback DM (2000) Hypocalcemia: diagnosis and treatment. In: Feingold KR, Anawalt B, Boyce A et al (eds) Endotext [Internet]. MDText.com, Inc., South Dartmouth. PMID: 25905251

4. Kelly A, Levine MA (2013) Hypocalcemia in the critically ill patient. J Intensive Care Med 28:166-177

5. Rubin MR (2020) Recent advances in understanding and managing hypoparathyroidism. F1000Res 9:F1000 